\title{
Arcing-based protection system for the klystron output window of the BEPCII Linac
}

\author{
Xiang He ${ }^{1} \mathbb{D} \cdot$ Qi Le $^{1} \cdot$ Lei Qian $^{1} \cdot$ Bing-Lin Deng ${ }^{1} \cdot$ Jing-Ru Zhang $^{1}$
}

Received: 10 March 2020 / Revised: 20 April 2020 / Accepted: 27 April 2020 / Published online: 11 May 2020

(c) The Author(s) 2020

\begin{abstract}
Purpose The Beijing Electron-Positron Collider II (BEPCII) Linac is powered by 20 klystrons, and the output windows of each klystron need to be protected from being broken, which are currently protected by a system based on reflected radiofrequency (RF) power and vacuum levels. The primary weak point of reflection-based protection is the difficulty of accurately measuring the value caused by an uneven waveform. Moreover, vacuum protection is restricted by the long response time, which results in delayed protection. Both of the weak points may damage the output window of the klystron. An arcing-based protection system is then introduced into the BEPCII Linac, considering the constant presence of light during arcing and the experiences of other laboratories and colleagues in the RF group.

Methods The system development mainly consists of the design and test of the E-bend waveguide observation window, selection of the arcing detection module, offline joint test, system assembling and online operation.

Results The system was assembled with a new klystron at the K6 position on the summer of 2018. During the following annual operation year, the light signal was captured, and the interlock was successfully realized.

Conclusion The arcing-based protection system assembled online was found to work well. Moreover, the light signal was successfully captured, and the interlock function worked fine. The system is planned to be applied in other klystron locations in the future.
\end{abstract}

Keywords Arcing-based protection system $\cdot$ Klystron output window $\cdot$ E-bend waveguide observation window

PACS 29.20.-c

\section{Introduction}

The accelerating structures in the BEPCII Linac are powered by 20 klystrons [1]. The output window of the klystron is the key part of isolating the vacuum environment between inside and outside of a klystron, which will seriously affect the life of the klystron if broken. Therefore, effectively protecting the klystron output window from being broken has been a key subject in the last years.

Funded by Youth Innovation Promotion Association CAS (2020015).

Xiang $\mathrm{He}$

hexiang@ihep.ac.cn

1 Key Laboratory of Particle Acceleration Physics and Technology, Institute of High Energy Physics, Chinese Academy of Sciences, Beijing 100049, China
The conventional methods for protection include reflection-based [2] and vacuum protection [3]. The former is based on the measurement of the reflected RF power picked by a waveguide directional coupler and transmitted to the peak power meter [4]. If the measured reflected power value exceeds the protection threshold of $2 \mathrm{MW}$, the output level of the peak power meter will change, and the trigger of the klystron modulator will be turned off by the interlock box. Meanwhile, vacuum protection is based on the measured value of the vacuum meter or the vacuum pump current. Similar to the reflection-based protection, the trigger will be turned off if the value exceeds the protection threshold. However, both methods have their natural weak points.

Reflection-based protection relies on the accurate measurement of the reflected power and the fast response. The response time of the reflection-based protection can now reach several microseconds due to the application of the digital technology [2]. However, the accurate measurement 
of the power level is an important issue because the reflected power waveform is always uneven, with a few considerably high points. Therefore, balancing the number of the sampling points and the algorithm is difficult when the reflected power is being measured. Excessive sampling points may slow down the calculation and miss the instantaneous reflection surge. On the contrary, very few points may miss the reflection power peak. The operation becomes unstable if the interlock works too often just because of several points above the threshold (assuming now that the average reflected power is far below the threshold within the pulse width). For the newly installed klystron and waveguide, the output power of the klystron lower than $2 \mathrm{MW}$ is also the gassing out point. The output window may be damaged, but the $2 \mathrm{MW}$ protection threshold cannot be reached even if the total power is reflected. The secondary electron multiplication effect in the low power range is also a great threat to the output window, which cannot be protected by the reflectionbased protection.

Vacuum protection is relatively more accurate. However, from gassing to interlock protection, which undergoes value changing of the vacuum gauge or pump current, and data transmission takes a long process. Consequently, the output window may be damaged because of the delayed response.

When arcing happens, the vacuum deterioration and reflection increase, and the light signal generation always simultaneously occurs, among which the light signal is the fastest for detection, transmission, and processing. Therefore, the arcing-based protection system is introduced into the BEPCII Linac according to experiences of other laboratories [5] and our colleagues in the RF group.

\section{System design and off-line test}

Figure 1 illustrates the arcing-based protection system. The E-bend waveguide observation window is connected to the klystron output waveguide. The observation window is facing to the klystron output window with a hole on the wide wall of the E-bend waveguide. The vacuum is sealed by using a special type of glass with a ConFlat flange.

An optical fiber is connected to the observation window by a structure designed for totally blocking outside light. The optical fiber has a strong light-concentrating structure at the end, enabling it to detect light higher than 2 lx. The arcing detection module will give an output signal within $7 \mu$ s according to the brightness. Considering the whole process of light detection, transmission, and processing, the estimated total time from the arcing light generation to trigger tuning off should be less than $50 \mu$ s. The highest pulse repetition rate of the BEPCII Linac is $50 \mathrm{~Hz}(20 \mathrm{~ms})$; hence, the arcing-based protection system can turn off the trigger before the arrival of the next pulse, thereby protecting the

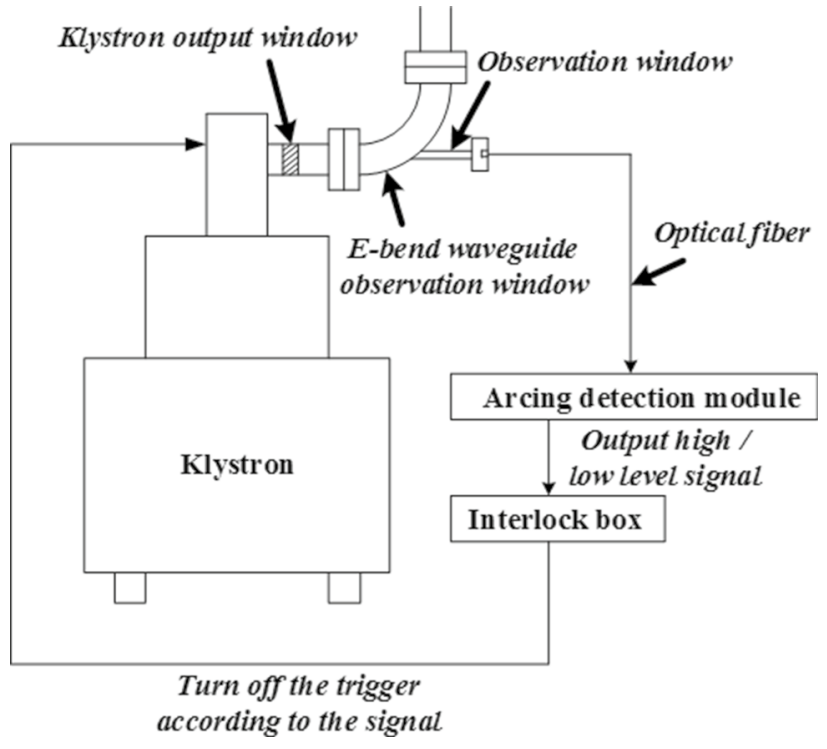

Fig. 1 Schematic of the arcing-based protection system

klystron output window from being broken. The light signal is a direct physical quantity; thus, compared with the reflection-based and vacuum protection, the arcing-based protection system has the advantages of direct and accurate measurement and fast response time.

The system design and the off-line test mainly consist of the design and test of the E-bend waveguide observation window, selection of the arcing detection module, and offline joint test.

\section{Design and test of the E-bend waveguide observation window}

The traditional E-bend waveguide for BEPCII is a $90^{\circ}$ bend with a sectional dimension of $144 * 144 \mathrm{~mm}$ (shown in the middle of Fig. 2). The female flange is the input port, while the male flange is the output. The arcing phenomenon on the output window can be observed by adding an observation window, which is vertical to the female flange. The E-bend waveguide observation window is shown on the top of Fig. 2.

The E-bend waveguide observation window was simulated by using the FEA (finite element analysis) software CST (computer simulation technology) [6]. The input voltage standing wave ratio (VSWR), insertion loss (IL), leaked field at the observation window, and the maximal electric field inside the structure were considered. The simulation model is shown at the bottom of Fig. 2. We defined port 1 as the input port, port 2 as the output port, and port 3 as the observation window. 

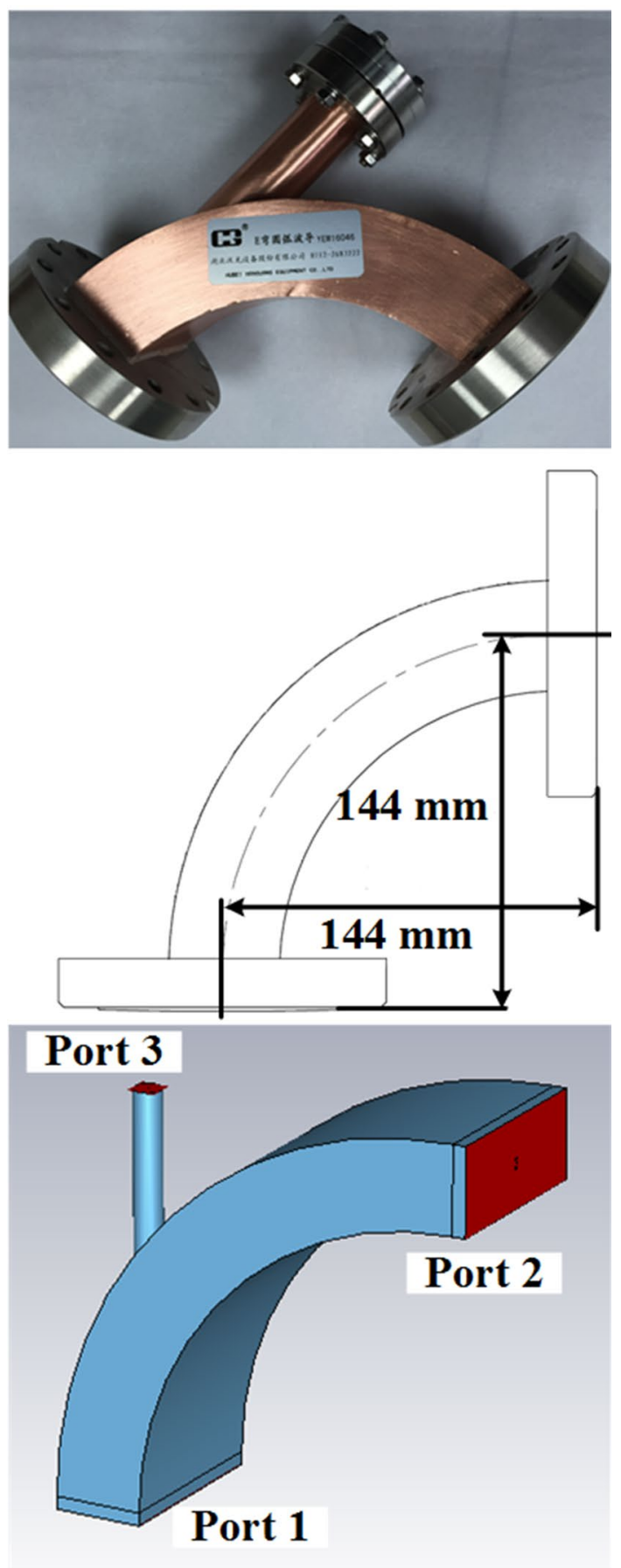

Fig. 2 Prototype (top) and simulation model (bottom) of the E-bend waveguide observation window, brief drawing of the traditional E-bend waveguide (middle)

The frequency response of S11 (VSWR) and S31 (i.e., the leaked filed, also known as the isolation) is presented at the top inset of Fig. 3, whereas the electric field distribution is depicted at the bottom. The simulation results at the central frequency of $2856 \mathrm{MHz}$ are as follows: $\mathrm{S} 11$ was $-35.25 \mathrm{~dB}$ (VSWR: 1.035), IL (S21) was $-0.076 \mathrm{~dB}$, and isolation
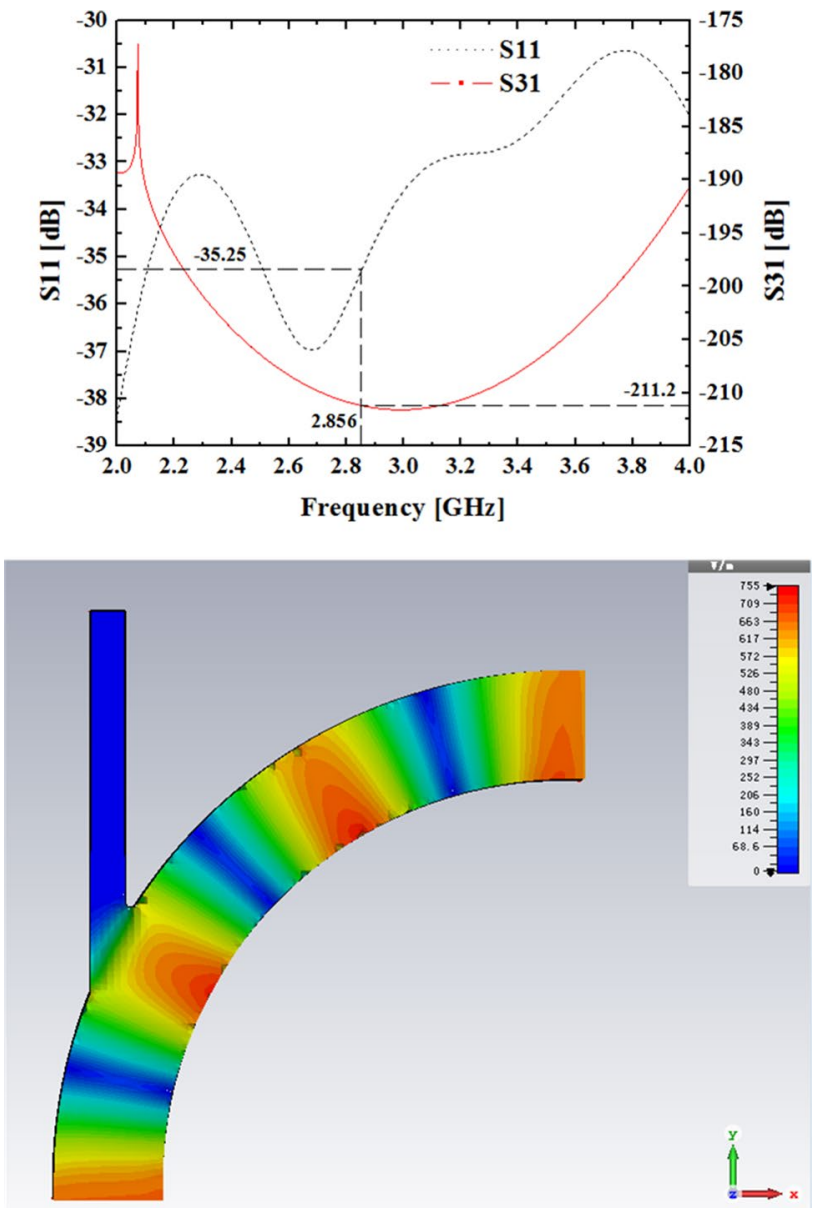

Fig. 3 Frequency response of S11 and S31 (top) and electric field distribution (bottom) of the E-bend waveguide observation window

(S31) was $-211.2 \mathrm{~dB}$. The electric field distribution also showed that much of the electric field transmitted inside the waveguide (red), while a few leaked from port 3 (dark blue) because of the microwave cutoff effect.

The isolation of $-211.2 \mathrm{~dB}$ means that the leaked field from port 3 was only in femtowatt $\left(10^{-15} \mathrm{~W}\right)$ for a $50 \mathrm{MW}$ input power, which can be neglected.

The structure was also chamfered at the connection between the window and the waveguide wall to reduce the maximum electric field value inside and avoid breakdown. The quantitative simulation showed that with a maximum input power of $50 \mathrm{MW}$, the maximum electric field in the waveguide was $15.5 \mathrm{MV} / \mathrm{m}$, which is much smaller than the breakdown field of $45.8 \mathrm{MV} / \mathrm{m}$ of the S-band high vacuum $\mathrm{RF}$ structure. This result indicates that no risk of breakdown exists.

The measurement and simulation results at $2856 \mathrm{MHz}$ are as follows: S11: $-28.09 /-35.25 \mathrm{~dB}$ (corresponding to the VSWR of $1.082 / 1.035)$ and IL: $-0.15 /-0.076 \mathrm{~dB}$. The measurement results were slightly worse than the simulation. 
However, they met the requirements, and the prototype worked stably online after installation.
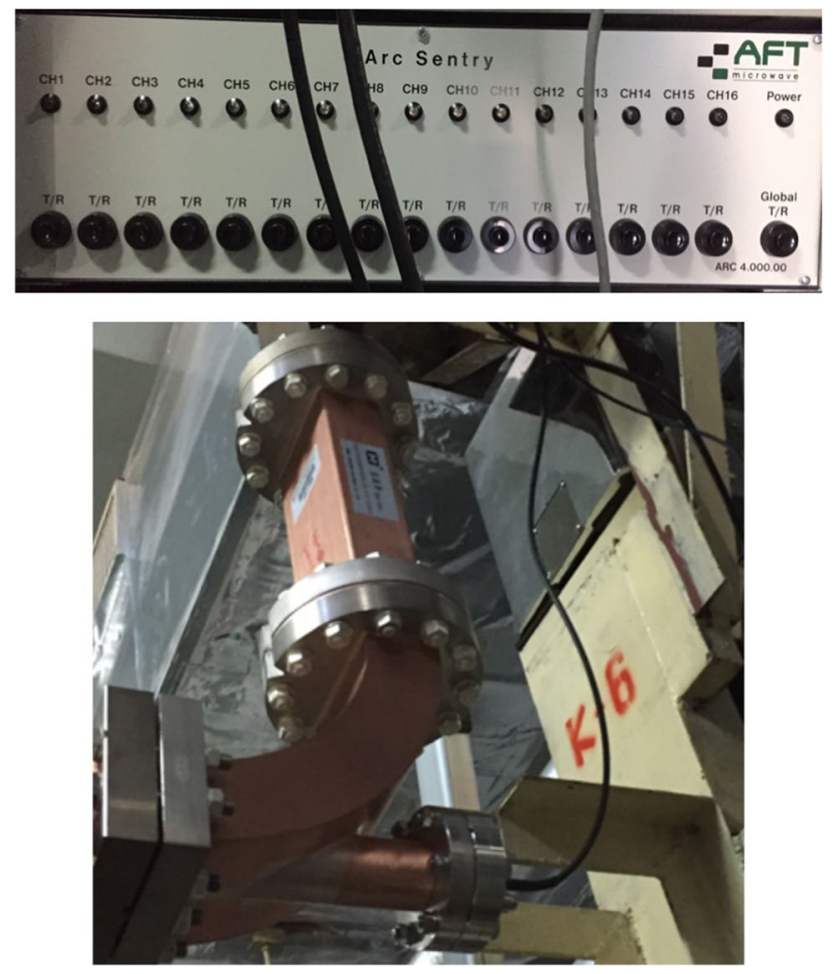

Fig. 4 Installed arcing detection module (top) and E-bend waveguide observation window with optical fiber (bottom)

\section{Selection of the arcing detection module and the off-line joint test}

The ARC4 type of German AFT Company was adopted as the arcing detection module, composed of a cabinet, an arcing detection board, and an optical fiber.

The off-line joint test was conducted after the fabrication and the measurement of the E-bend waveguide observation window. The arcing detection module can output a high- or low-level signal from the BNC port; the signal amplitude is proportional to the brightness of the captured light, which can be used as the input signal of the interlock box to protect the klystron output window.

\section{System assembling and online operation}

In the summer of 2018, the arcing-based protection system was assembled at the K6 position of the BEPCII Linac with a new klystron. The structure on the observation window could fix the fiber connector and block the light from outside. Simultaneously, the arcing detection module was installed in the cabinet of K6. Figure 4 shows the installed arcing detection module (top) and the E-bend waveguide observation window with an optical fiber (bottom).

The system worked stably during the following annual operation year. The light signal was successfully captured, and the interlock worked well. Figure 5 shows an

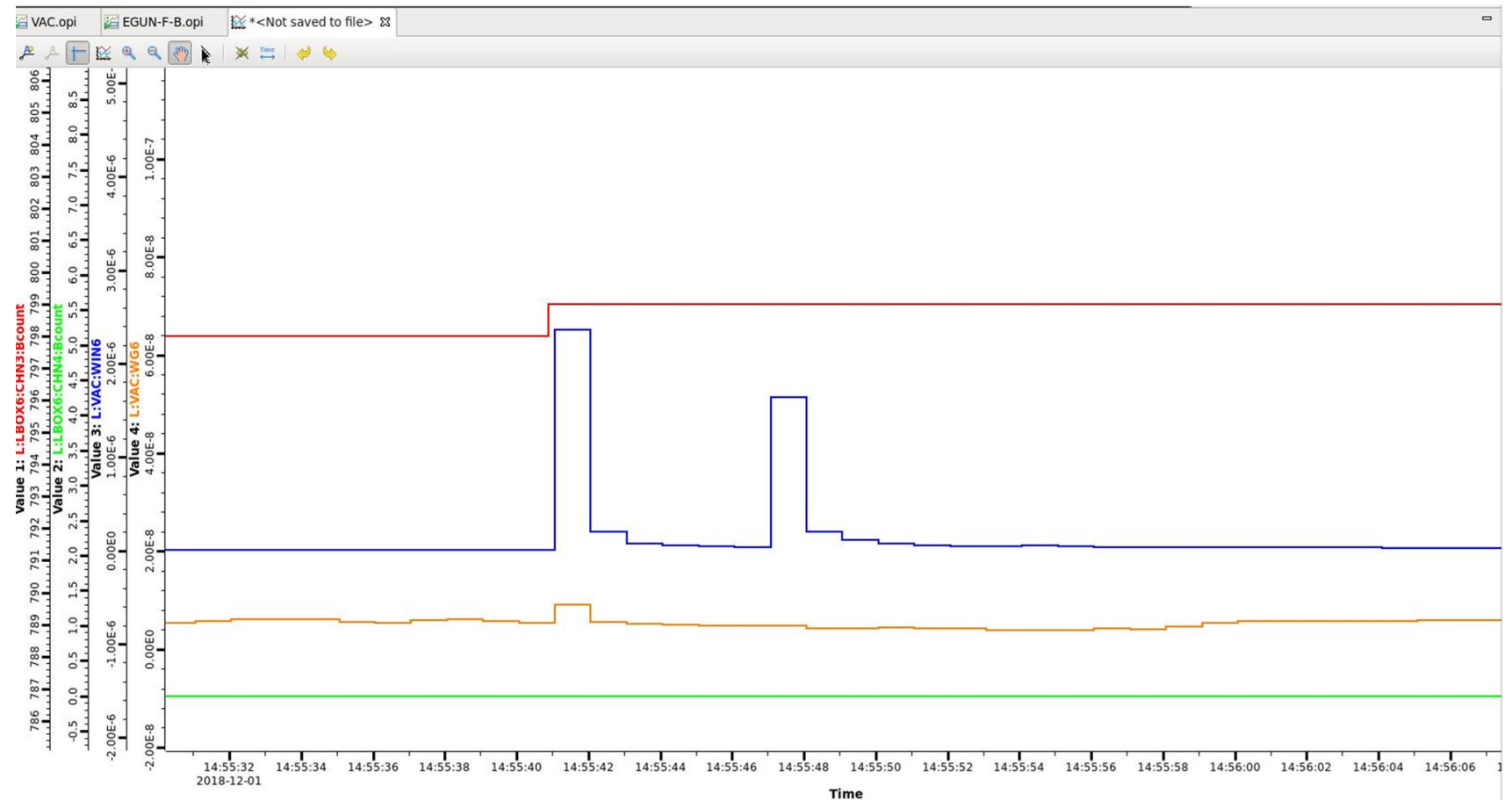

Fig. 5 Captured arcing light signal and vacuum change in December 2018 
arcing-based protection in December of 2018, in which the red (highest) curve denotes the count of the arcing numbers, and the blue (second highest) curve represents the vacuum value. The arcing-based protection system very clearly worked (i.e., the counted number of arcing increased by one in the curve) when the vacuum worsened (i.e., two peaks in the curve). Figure 5 also illustrates that the arcing-based protection is faster than the vacuum protection. However, the second vacuum protection (second peak in blue curve) had no detected arcing (no step in red curve), which means that the reflection-based and vacuum protection are still needed in spite of the advantages of the arcing-based protection system. Because if no arcing happens when the vacuum deteriorates, the reflection-based and vacuum protection are necessary for preventing the klystron output window being broken.

\section{Conclusions}

In this study, the arcing-based protection system assembled online was found to work well. Moreover, the light signal was successfully captured, and the interlock function worked fine. The system is expected to be applied to other klystron locations in the future.

Acknowledgements We would like to thank Editage (www.editage.cn) for English language editing.
Open Access This article is licensed under a Creative Commons Attribution 4.0 International License, which permits use, sharing, adaptation, distribution and reproduction in any medium or format, as long as you give appropriate credit to the original author(s) and the source, provide a link to the Creative Commons licence, and indicate if changes were made. The images or other third party material in this article are included in the article's Creative Commons licence, unless indicated otherwise in a credit line to the material. If material is not included in the article's Creative Commons licence and your intended use is not permitted by statutory regulation or exceeds the permitted use, you will need to obtain permission directly from the copyright holder. To view a copy of this licence, visit http://creativecommons.org/licenses/by/4.0/.

\section{References}

1. G.X. Pei, Y.L. Sun, Y.L. Chi, S.H. Wang, Progress of the injector Linac upgrade for the BEPC II project. High Energy Phys. Nuclear Phys. 28(11), 1214-1218 (2004)

2. X.J. Wang, J.R. Zhang, S.L. Pei, X. He, F.L. Zhao, Reflection protection of BEPCII linear accelerator klystron. High Power Laser Particle Beams 31(02), 31025101 (2019). (in Chinese)

3. M. Hou, Z.S. Zhou, RF power test and conditioning of the new accelerating structures for the BEPCII Linac. High Energy Phys. Nuclear Phys. 29(1), 81-85 (2005)

4. X.J. Wang, F.L. Zhao, X. He, J.R. Zhang, D. Dong, Development of peak power meter based on logarithmic detector. High Power Laser Particle Beams. 31(08), 085104 (2019). (in Chinese)

5. R. Tighe, ARC detection and interlock module for the PEP-II low level RF system, in Proceedings of PAC1997, pp. 2520-2522 (1997)

6. CST Microwave Studio, Computer Simulation Technology. www. cst.com 